\title{
Toeplitz Operators and Division Theorems in Anisotropic Spaces of Holomorphic Functions in the Polydisc
}

\author{
A. V. Harutyunyan ${ }^{1}$ \\ Yerewan State University
}

\begin{abstract}
This work is an introduction to anisotropic spaces, which have an $\omega$ weight of analytic functions and are generalizations of Lipshitz classes in the polydisc. We prove that these classes form an algebra and are invariant with respect to monomial multiplication. These classes are described in terms of derivatives. It is established that Toeplitz operators are bounded in these (Lipshitz and Djrbashian) spaces. As an application, we show a theorem about the division by good-inner functions in the mentioned classes is proved.
\end{abstract}

Key words and phrases: Toeplitz operators, anisotropic spaces, polydisc, goodinner function

2000 Mathematics Subject Classification: 35J40, 47G30, 58J32.

\footnotetext{
${ }^{1}$ Supported by the Deutscher Akademischer Austauschdienst (DAAD)
} 


\section{Contents}

1 Introduction 3

2 The classes $\widetilde{\Lambda}\left(\omega_{1}, \ldots, \omega_{n}\right)$ and basic constructions 4

3 Main results $\quad 9$

4 Toeplitz Operators in classes $\widetilde{\Lambda}\left(\omega_{1}, \ldots, \omega_{n}\right) \quad 14$

5 Linear Continuous Functionals on $\mathbb{H}^{p}(\omega) 0<p \leq 1$ and Ap$\begin{array}{ll}\text { plications } & 17\end{array}$ 


\section{Introduction}

The significance of factorization into outer and inner functions in the theory of Hardy classes and their applications is well known. As was shown in 1971 by Korenblum [1], V. P. Khavin [2] and F. A. Shamoian [3], such a factorization can also be successfully used to investigate several classes of functions which are holomorphic in the disk and smooth up to its boundary. Their results were based on the invariance of most such classes under the action of Toeplitz operators of the form $T_{h}(f)=P_{+}(f \cdot h)$, where $h$ is an arbitrary bounded, holomorphic function in the disk, and $P_{+}$is the Riesz' projector.

These operators play important role not only for investigated questions of factorization but also in investigations of closed ideals in algebras of analytic functions, questions of the best approximation with rational functions etc.(see [4],[5]).

This paper focuses an anisotropic classes of Lipshitz type. It is verified that these classes are invariant with respect to multiplication by monomials, they make an algebra in differ to early known polymetric Lipshitz classes. The investigation of Toeplitz operators in the polydisc is of special interest. The polydisc is a product of $n$ discs in the one dimensional complex plane, and one would expect that natural generalizations of results from the onedimensional setting would be valid here. But research has shown, that this is not so. The case of a polydisc is different from the $n=1$ case and the case of an $n$-dimensional sphere. For example, let's consider the classical theorem of Privalov: If $f \in \operatorname{Lip} \alpha$, then $K f \in \operatorname{Lip} \alpha$, where $K f$ is a Cauchy type integral. It is known that the analogue of this theorem for known multidimensional Lipshitz classes is not true ([6]), even though the analogue of this theorem for a sphere is valid ([7]). Since the polydisc is the natural generalization of a circle, the analogue of this theorem should also hold for a polydisc, but in a new generalization of Lipshitz classes. That had yet to be identified. Related to this generalization was also the description of the multidimensional analogues of M. Djrbashian classes. In a 1993 work [8], new Lipshitc classes were introduced for both the noninteger and integer indices; these newly introduced classes allowed one to answer the above questions.

In Shamoians work [9] Djrbashian classes were a generalized for an $\omega$ weight, as well as the forms of the linearly continuous functions in those classes were given. The description of the conjugate of weighted classes $\mathbb{H}^{p}(\omega)$ in terms of smooth functions (as it was done by introducing new Lipshitz classes in [8]) raised a new problem. The current work is related to the problem of introducing $\omega$ - weighted Lipshitz classes and their properties.

In Sektion 1 these classes are defined and two lemmas are proved, which are then used for proving the main theorems. In Section 2 theorems are proved that describe the properties of the introduced Lipshitz classes: in 
particular the description of these classes in terms of derivatives, their forming an algebra, and their invariance with respect to monomial multiplication. We would like to mention that the previously known classes do not have the last two properties. In Section 3 we consider Toeplitz operators in these classes and discuss their boundedness. Here a theorem is also proved about the division by suitable (good-inner) functions in these new and Djbaschian classes.

Acknowledgement: The author would like to thank DAAD (Deutscher Akademischer Austauschdienst) for financial support and the opportunity of conducting research in Germany.

Special thanks go to Prof. B.- W. Schulze ( Universität Potsdam, Institut für Mathematik) for helpful comments, suggestions and the opportunity of working in his group and participating in research seminars.

\section{The classes $\widetilde{\Lambda}\left(\omega_{1}, \ldots, \omega_{n}\right)$ and basic constructions}

Denote by $\mathbb{U}^{n}=\left\{z=\left(z_{1}, \ldots, z_{n}\right) \in \mathbb{C}^{n},\left|z_{j}\right|<1,1 \leq j \leq n\right\}$ the unit polydisk in the $n$-dimensional complex plane $\mathbb{C}^{n}$, by $\mathbb{T}^{n}=\left\{z=\left(z_{1}, \ldots, z_{n}\right) \in\right.$ $\left.\mathbb{C}^{n},\left|z_{i}\right|=1,1 \leq i \leq n\right\}$ its torus, and by $\mathbb{H}\left(\mathbb{U}^{n}\right)$ the set of holomorphic functions in $\mathbb{U}^{n}$, by $\mathbb{H}^{\infty}\left(\mathbb{U}^{n}\right)$ the set of bounded holomorphic functions in $\mathbb{U}^{n}$. In what follows we write $\mathbb{Z}_{+}^{k}$ for the set of vectors, whose components are natural numbers and $\mathbb{R}_{+}^{k}$ for the set of vectors with positive components.

Let $\omega_{j} \geq 0, \omega_{j}(t) \rightarrow 0(t \rightarrow 0), 1 \leq j \leq n$ be a nonegative measurable function on $(0,1)$, such that $\omega_{j}(t) \cdot t^{\alpha}$ increases on $(0, a]$ for some $\alpha<1$ and $\omega_{j}(t) / t^{\beta}$ decreases on $[a, 1)$ for some $\beta<1$ and for $a \in(0,1)$.

Denote by $\Omega$ the class of functions $\omega$ with this property.

Let $f(\zeta), \zeta=\left(\zeta_{1}, \ldots, \zeta_{n}\right) \in \mathbb{T}^{n}$ be a measurable bounded function, and $\omega=\left(\omega_{1}, \ldots, \omega_{n}\right)$, where $\omega_{j} \in \Omega$. Then $\omega_{j}(t)=O\left(t^{\gamma_{j}}\right), 0<\gamma_{j}<1$, for $t \rightarrow+0,0 \leq j \leq n$.

Definition 2.1 A function $f$ is said to be in $\widetilde{\Lambda}(\omega)$, if for all

$$
\begin{gathered}
(k)=\left(i_{1}, \ldots, i_{k}\right), 1 \leq k \leq n, 1 \leq i_{j} \leq n, 1 \leq j \leq n, \\
h=\left(h_{1}, \ldots, h_{n}\right) \in \mathbb{R}^{n} \text { satisfies } \\
\left|\Delta_{h_{i_{1}}, \ldots, h_{i_{k}}} f(\zeta)\right| \leq C_{(k)}(f) \omega_{i_{1}}\left(\left|h_{i_{1}}\right|\right) \ldots \omega_{i_{k}}\left(\left|h_{i_{k}}\right|\right),
\end{gathered}
$$

where

$$
\Delta_{h_{i_{1}}, \ldots, h_{i_{k}}}=\Delta_{h_{i_{1}}}\left(\ldots\left(\Delta_{h_{i_{k}}}\right)\right)
$$

The smallest constant $C_{(k)}(f)$ satisfying the last inequality is 


$$
\sup _{\substack{\left(\vartheta_{1} \ldots, \vartheta_{n}\right) \in \mathbb{Q}^{n} \\\left(\left|h_{i_{1}}\right|, \ldots,\left|h_{i_{k}}\right|\right) \in \mathbb{R}_{+}^{k}}} \frac{\left|\Delta_{h_{i_{k}} \ldots h_{i_{1}}} f\left(e^{i \vartheta_{1}}, \ldots, e^{i \vartheta_{n}}\right)\right|}{\omega\left(\left|h_{i_{1}}\right|\right) \cdots \omega\left(\left|h_{i_{k}}\right|\right.}=C_{(k)}(f)<+\infty,
$$

We set

$$
\|f\|_{\widetilde{\Lambda}^{a}(\omega)}=\max _{1 \leq k \leq n} \max _{1 \leq i_{1}<\cdots<i_{k} \leq k} C_{(k)}(f)+\|f\|_{\mathbb{H}^{\infty}} .
$$

Note that the classes $\widetilde{\Lambda}^{a}(\omega)$, for $\omega_{j}(t)=t_{j}^{\alpha_{j}}, j=1, \ldots, n ;\left(i_{1}, \ldots, i_{k}\right)=$ $(1, \ldots, n)$ were first introduced by S. M. Nikol'skii (see [10]).

Corollary 2.2 Let $\gamma_{j}>1,(1 \leq j \leq n)$ be noninteger. We set

$$
m_{j}=\left[\gamma_{j}\right], 1 \leq j \leq n,
$$

and

$$
F\left(e^{i \theta}, \cdots, e^{i \theta}\right)=\frac{\partial^{m} f\left(e^{i \theta_{1}}, \cdots, e^{\theta_{n}}\right)}{\partial \theta^{m_{1}} \cdots \partial \theta^{m_{n}}} .
$$

Then $\widetilde{\Lambda}^{a}(\omega)$ consists of all $f$, for which $F \in \widetilde{\Lambda}^{a}\left(\omega / t^{m}\right)$.

For a description of these classes in terms of derivatives, we must first prove the following lemma which in the case of $\omega(t)=t^{\alpha}$ is a consequence of the Hardy-Littlewood theorem (see [11]).

Lemma 2.3 Let $\left|f^{\prime}(z)\right| \leq A \cdot \omega(1-|z|) \cdot(1-|z|)^{-1}, z \in \mathbb{U}$, then

$$
|f(z+h)-f(z)| \leq 5 \cdot A(\alpha) \cdot \omega(|h|), z, z+h \in \overline{\mathbb{U}} .
$$

And conversely, if $f \in \mathbb{H}(\mathbb{U})$ and $|f(z+h)-f(z)| \leq B \cdot \omega(|h|)$, then

$$
\left|f^{\prime}(z)\right| \leq B \omega(1-|z|)(1-|z|)^{-1} .
$$

Proof. It suffices to prove the lemma for the case $h>0$. We have

$$
f\left(e^{i \theta+i h}\right)-f\left(e^{i \theta}\right)=\int_{\theta}^{\theta+h} f^{\prime}\left(e^{i t}\right) d e^{i t}=\int_{I_{1}}+\int_{I_{2}}+\int_{I_{3}},
$$

where $I_{1}$ is the segment $1 \geq r \geq 1-h$ of the line $\arg z=\theta ; I_{2}$ is arc $z=(1-h) e^{i t}, \theta \leq t \leq \theta+h$, and $I_{3}$ is the segment $1-h \leq r \leq 1$ of the line $\arg z=\theta+h$. We now estimate every integral separately.

The estimate of $\int_{I_{1}}$ : 


$$
\begin{aligned}
\left|\int_{I_{1}} f^{\prime}\left(e^{i t}\right) d e^{i t}\right| & \leq \int_{1-h}^{1}\left|f^{\prime}\left(\rho e^{i \theta}\right)\right| d \rho \leq A \cdot \int_{1-h}^{1} \frac{\omega(1-\rho)}{1-\rho} d \rho \\
& =A \cdot \int_{0}^{h} \frac{\omega(u)}{u} d u=A\left\{\int_{0}^{\delta} \frac{\omega(u)}{u} d u+\int_{\delta}^{h} \frac{\omega(u)}{u} d u\right\} \\
& \leq A\left\{\int_{0}^{\delta} \frac{1}{u^{1-\alpha} d t}+\int_{\delta}^{h} \frac{\omega(u) \cdot u^{\alpha}}{u^{\alpha+1}} d t\right\} \\
& \leq \delta / \alpha+\omega(h)<2 A \cdot \omega(h) .
\end{aligned}
$$

We use the fact that $\omega(h) / h^{\alpha}$ increases for some $\alpha>0$.

The estimate of $\int_{I_{2}}$ :

$$
\left|f^{\prime}\left(e^{i t}\right) d e^{i t}\right|=\left|\int_{\theta}^{\theta+h} f^{\prime}\left((1-h) e^{i \phi}\right) d e^{i \phi}\right| \leq \int_{\theta}^{\theta+h} \frac{\omega(h)}{h}=\omega(h) .
$$

$\int_{I_{3}}$ can be estimated similarily to $\int_{I_{1}}$. Finally,

$$
\left|f\left(e^{i \theta+i h}\right)-f\left(e^{i \theta}\right)\right| \leq 5 A(\alpha) \omega(h) .
$$

Conversely, as $f \in \mathbb{H}$, then

$$
f(z)=\frac{1}{2 \pi i} \int_{\mathbb{T}} \frac{f(\zeta)}{\zeta-z} d \zeta
$$

where $z=r e^{i \phi}$.

Differentiating, we obtain

$$
\begin{aligned}
f^{\prime}(z) & =\frac{1}{2 \pi i} \int_{\mathbb{T}} \frac{f(\zeta)}{(\zeta-z)^{2}} d \zeta=\frac{i}{2 \pi i} \int_{\mathbb{T}} \frac{f\left(e^{i \theta}\right) e^{i \theta}}{\left(e^{i \theta}-r e^{i \phi}\right)^{2}} d \theta \\
& =\frac{1}{2 \pi} \int_{\mathbb{T}} \frac{f\left(e^{i \theta}\right) e^{i \theta}}{e^{2 i \phi}\left(e^{i(\theta-\phi)}-r\right)^{2}} d \theta=\frac{e^{-i \phi}}{2 \pi} \int_{\mathbb{T}} \frac{f\left(e^{i \theta}\right) e^{i \theta-i \phi}}{\left(e^{i \theta-i \phi}-r\right)^{2}} d \theta \\
& =\frac{e^{-i \phi}}{2 \pi i} \int_{\mathbb{T}} \frac{f\left(e^{i t+i \phi}\right)}{\left(e^{i t}-r\right)^{2}} d e^{i t}
\end{aligned}
$$

for $t=\theta-\phi$.

As

$$
\frac{1}{2 \pi i} \int \frac{1}{(\zeta-z)^{2}} d \zeta=0
$$


it follows that

$$
\begin{aligned}
\left|f^{\prime}(z)\right| & =\left|\frac{e^{-i \phi}}{2 \pi i} \int_{\mathbb{T}} \frac{f\left(e^{i t+i \phi}\right)-f\left(e^{i \phi}\right)}{\left(e^{i t}-r\right)^{2}} d e^{i t}\right| \\
& \leq \frac{1}{2 \pi} \int_{\mathbb{T}} \frac{\left|\Delta_{t} f\left(e^{i t}\right)\right|}{\left|e^{i t}-r\right|^{2}} d|t| \leq \frac{1}{2 \pi} \int_{-\pi}^{\pi} \frac{\omega(|t|)}{\left|e^{i t}-r\right|^{2}} d|t| .
\end{aligned}
$$

We have

$$
\left|e^{i t}-r\right|^{2}=(1-r)^{2}+4 r \sin ^{2} t / 2, \sin t>\frac{2}{\pi} t ;-\pi / 2<t<\pi / 2 .
$$

From this we get

$$
\begin{aligned}
\int_{-\pi}^{\pi} \frac{\omega(|t|) d t}{\left|e^{i t}-r\right|^{2}} & \leq \int_{-\pi}^{\pi} \frac{\omega(|t|) d t}{(1-r)^{2}+4 r t^{2} / \pi^{2}}=2 \cdot \int_{0}^{\pi} \frac{\omega(|t|) d t}{(1-r)^{2}+4 r t^{2} / \pi^{2}} \\
& =2 \cdot\left\{\int_{0}^{1-r} \frac{\omega(t) d t}{(1-r)^{2}+4 r t^{2} / \pi^{2}}+\int_{1-r}^{\pi} \frac{\omega(t) d t}{(1-r)^{2}+4 r t^{2} / \pi^{2}}\right\} .
\end{aligned}
$$

Since $\omega(t) \cdot t^{\alpha}$ increases, then for the first integral we obtain

$$
\begin{gathered}
\int_{0}^{1-r} \frac{\omega(t) t^{\alpha} d t}{(1-r)^{2}+4 r t^{2} / \pi^{2}} \leq \omega(1-r) \cdot(1-r)^{\alpha} \int_{0}^{1-r} \frac{1}{t^{\alpha}} d t=\frac{\omega(1-r)}{1-r} \\
(\alpha<1) .
\end{gathered}
$$

Let us now estimate the second integral. Setting $t=(1-r) u$, we get

$$
\begin{aligned}
& \frac{1}{(1-r)^{2}} \int_{1}^{\pi / 1-r} \frac{\omega((1-r) u)(1-r) d u}{1+4 r u^{2} / \pi^{2}} \\
= & \frac{1}{(1-r)} \int_{1}^{\pi / 1-r} \frac{\omega((1-r) u) d u}{1+4 r u^{2} / \pi^{2}} \\
= & \frac{1}{1-r} \int_{1}^{+\infty} \frac{\omega((1-r) u) d u}{1+4 r u^{2} / \pi^{2}} \\
= & \frac{1}{1-r} \int_{1}^{+\infty} \frac{\omega((1-r) u)((1-r) u)^{\beta}}{\left(1+4 r u^{2} / \pi^{2}\right)((1-r) u)^{\beta}} d u \\
< & \frac{1}{1-r} \frac{\omega(1-r)(1-r)^{\beta}}{(1-r)^{\beta}} \int_{1}^{+\infty} \frac{d u}{u^{\beta}\left(1+4 r u^{2} / \pi^{2}\right)} .
\end{aligned}
$$


Here we have used the property that $\omega(t) / t^{\beta}$ decreases on $[a, 1]$. Outside the Interval $(0,1)$ we consider the periode extension of $\omega_{j}, 1 \leq j \leq n$.

The integral

$$
\int_{1}^{+\infty} \frac{d u}{u^{\beta}\left(1+4 r u^{2} / \pi^{2}\right)}
$$

converges for $\beta<1$, hence we get the desired estimate.

The following lemma will be used in the proof of our theorem.

Lemma 2.4 If $h \in \mathbb{H}(\mathbb{U})$, then

$$
\frac{1}{2 \pi i} \int_{\mathbb{T}} \frac{\overline{h(\zeta)}}{(\zeta-z)^{2}} d \zeta=0,|z|<1
$$

Proof. As $h_{z} \in \mathbb{H}(\mathbb{U})\left(h_{z}(\zeta)=h \zeta(1-\bar{z} \zeta)^{-2}\right)$, we get

$$
\begin{aligned}
& \frac{1}{2 \pi i} \int_{\mathbb{T}} \frac{h \overline{(\zeta})}{(\zeta-z)^{2}} d \zeta=\frac{1}{2 \pi i} \overline{\int_{\mathbb{T}} \frac{h(\zeta)}{(\bar{\zeta}-\bar{z})^{2}} d \bar{\zeta}}= \\
= & \frac{1}{2 \pi i} \overline{\int_{\mathbb{T}} \frac{h(\zeta) i \bar{\zeta}}{\left(\bar{\zeta}^{2}(1-\bar{z} \zeta)^{2}\right.} d \zeta}=\overline{\frac{1}{2 \pi} \int_{\mathbb{T}} \frac{h(\zeta) \zeta}{(1-\bar{z} \zeta)^{2}} d \zeta}=0 .
\end{aligned}
$$

In case some $\gamma_{j}$ are integral we obtain analogs of Zigmund classes. This case is breated in much the same way as the case of nonintegral $\gamma_{j}$. So, restrict ourselves to the definition of these classes and formulation of the corresponding theorem (see [8]).

Let $\gamma_{j}$ be equal to $=1$ for $1 \leq j \leq n$.

Definition 2.5 A function $f$ is said to be in $\widetilde{\Lambda}_{*}(\omega)$, if for all

$$
\begin{gathered}
(k)=\left(i_{1}, \ldots, i_{k}\right), 1 \leq k \leq n, 1 \leq i_{j} \leq n, \\
1 \leq j \leq n, h=\left(h_{1}, \ldots, h_{n}\right) \in \mathbb{R}^{n}
\end{gathered}
$$

it fulfils

$$
\left|\Delta_{h_{i_{1}}, \ldots, h_{i_{k}}}^{*} f(\zeta)\right| \leq C_{(k)}(f) \omega_{i_{1}}\left(\left|h_{i_{1}}\right|\right) \ldots \omega_{i_{k}}\left(\left|h_{i_{k}}\right|\right),
$$

where

$$
\Delta_{h_{i_{1}}, \ldots, h_{i_{k}}}^{*}=\Delta_{h_{i_{1}}}^{*}\left(\ldots\left(\Delta_{h_{i_{k}}}^{*}\right)\right)
$$

and

$$
\begin{gathered}
\Delta_{h_{j}}^{*} f\left(e^{\vartheta}\right)=f\left(e^{i \vartheta_{1}}, \ldots, e^{i \vartheta_{j-1}}, e^{i\left(\vartheta_{j}+h_{j}\right)}, e^{i \vartheta_{j+1}}, \ldots, e^{i \vartheta_{n}}\right) \\
-2 \cdot f\left(e^{i \vartheta_{1}}, \ldots, e^{i \vartheta_{n}}\right)+f\left(e^{i \vartheta_{1}}, \ldots, e^{i \vartheta_{j-1}}, e^{i\left(\vartheta_{j}-h_{j}\right)} e^{i \vartheta_{j+1}}, \ldots, e^{i \vartheta_{n}}\right) .
\end{gathered}
$$


We set

$$
\|f\|_{\widetilde{\Lambda}_{*}^{a}(\omega)}=\max _{1 \leq k \leq n} \max _{1 \leq i_{1}<\cdots<i_{k} \leq k} C_{(k)}(f)+\|f\|_{\mathbb{H} \infty},
$$

where $C_{(k)}(f)$ is the smallest constant from (1).

\section{Main results}

Theorem 3.1 If $f \in \mathbb{H}^{\infty}\left(\mathbb{U}^{n}\right)$, then $f \in \widetilde{\Lambda}^{a}(\omega)$ if and only if

$$
\left|\frac{\partial^{k} f(z)}{\partial z_{i_{1}} \ldots z_{i_{k}}}\right| \leq C \cdot C_{(k)}(f) \cdot \prod_{j=1}^{k} \frac{\omega_{i_{j}}\left(1-\left|z_{i_{j}}\right|\right)}{1-\left|z_{i_{j}}\right|},
$$

where $1 \leq i_{k} \leq n, 1 \leq k \leq n$.

Proof. Let (2) be fulfilled. Denoting

$$
F\left(z_{i_{1}}\right)=\frac{\partial^{k-1} f(z)}{\partial z_{i_{2}} \ldots \partial z_{i_{k}}}
$$

we get

$$
\left|F^{\prime}\left(z_{i_{1}}\right)\right| \leq C \cdot C_{(k)}(f) \cdot \frac{\omega_{i_{1}}\left(1-\left|z_{i_{1}}\right|\right)}{1-\left|z_{i_{1}}\right|} .
$$

In view of Lemma (2.3)we obtain $F \in \operatorname{Lip}\left(\omega_{i_{1}}\right)$, i.e.,

$$
\left|\Delta_{h_{i_{1}}} F\left(\rho \zeta_{1}\right)\right| \leq 3 C\left(i_{2}, \ldots, i_{k}\right) \omega_{i_{1}}\left(\left|h_{i_{1}}\right|\right)
$$

Repeating these arguments we obtain

$$
\left|\Delta_{h_{(k)}} f(\rho \zeta)\right| \leq 3 C_{(k)}(f) \omega_{i_{1}}\left(\left|h_{i_{1}}\right|\right) \ldots \omega_{i_{k}}\left(\left|h_{i_{k}}\right|\right) .
$$

Letting $\rho \rightarrow 1$, we get

$$
\left|\Delta_{h_{(k)}} f(\zeta)\right| \leq 3 C_{(k)}(f) \omega_{i_{1}}\left(\left|h_{i_{1}}\right|\right) \ldots \omega_{i_{k}}\left(\left|h_{i_{k}}\right|\right)
$$

Conversely, for the simplicity let us consider the case $n=2$. Our goal is to estabilish estimates

$$
\begin{aligned}
& \left|\frac{\partial f(z)}{\partial z_{1}}\right| \leq C_{f} \frac{\omega_{1}\left(1-\left|z_{1}\right|\right)}{1-\left|z_{1}\right|} \\
& \left|\frac{\partial f(z)}{\partial z_{2}}\right| \leq C_{f} \frac{\omega_{2}\left(1-\left|z_{2}\right|\right)}{1-\left|z_{2}\right|}
\end{aligned}
$$




$$
\left|\frac{\partial^{2} f(z)}{\partial z_{1} \partial z^{2}}\right| \leq C_{f} \frac{\omega_{1}\left(1-\left|z_{1}\right|\right) \omega_{2}\left(1-\left|z_{2}\right|\right)}{\left(1-\left|z_{1}\right|\right)\left(1-\left|z_{2}\right|\right)} .
$$

Estimate (4): Using transformations of Lemma 2.3, we get:

$$
\frac{\partial f(z)}{\partial z_{1}}=\frac{e^{-i \phi_{1}}}{(2 \pi)^{2}} \int_{\mathbb{Q}^{2}} \frac{\Delta_{t_{1}} f\left(e^{i \phi_{1}}, e^{i \phi_{2}+i t_{2}}\right) e^{i t_{1}} e^{i t_{2}}}{\left(e^{i t_{1}}-r_{1}\right)^{2}\left(e^{i t_{2}}-r_{2}\right)} d t_{1} d t_{2} .
$$

Hence it follows that

$$
\begin{aligned}
\frac{\partial f(z)}{\partial z_{1}}= & \frac{e^{-i \phi_{1}}}{(2 \pi)^{2}}\left\{\int_{\mathbb{Q}^{2}} \frac{\left(\Delta_{t_{1}} f\left(e^{i \phi_{1}}, e^{i \phi_{2}+i t_{2}}\right)-\Delta_{t_{1}} f\left(e^{i \phi_{1}}, e^{i \phi_{2}}\right)\right) e^{i t_{1}} e^{i t_{2}}}{\left(e^{i t_{1}}-r_{1}\right)^{2}\left(e^{i t_{2}}-r_{2}\right)} d t_{1} d t_{2}\right. \\
& \left.+\int_{\mathbb{Q}^{2}} \frac{\Delta_{t_{1}} f\left(e^{i \phi_{1}}, e^{i \phi_{2}}\right) e^{i t_{1}} e^{i t_{2}}}{\left(e^{i t_{1}}-r_{1}\right)^{2}\left(e^{i t_{2}}-r_{2}\right)} d t_{1} d t_{2}\right\} \\
= & \frac{e^{-i \phi_{1}}}{(2 \pi)^{2}}\left\{\int_{\mathbb{Q}^{2}} \frac{\Delta_{t_{1} t_{2}} f\left(e^{i \phi_{1}}, e^{i \phi_{2}}\right) e^{i t_{1}} e^{i t_{2}}}{\left(e^{i t_{1}}-r_{1}\right)^{2}\left(e^{i t_{2}}-r_{2}\right)} d t_{1} d t_{2}\right. \\
& \left.+\int_{\mathbb{Q}^{2}} \frac{\Delta_{t_{1}} f\left(e^{i \phi_{1}}, e^{i \phi_{2}}\right) e^{i t_{1}} e^{i t_{2}}}{\left(e^{i t_{1}}-r_{1}\right)^{2}\left(e^{i t_{2}}-r_{2}\right)} d t_{1} d t_{2}\right\} .
\end{aligned}
$$

Then using also Lemma 2.3 we obtain:

$$
\begin{aligned}
\frac{\partial f(z)}{\partial z_{1}} & \leq C\left\{\int_{0}^{\pi} \int_{0}^{\pi} \frac{\omega_{1}\left(t_{1}\right) \omega_{2}\left(t_{2}\right) d t_{1} d t_{2}}{\left|e^{i t_{1}}-r_{1}\right|^{2}\left|e^{i t_{2}}-r_{2}\right|}\right. \\
& \left.+\int_{0}^{\pi} \frac{\omega_{1}\left(t_{1}\right) d t_{1}}{\left|e^{i t_{1}}-r_{1}\right|^{2}}\right\} \leq C \cdot \frac{\omega_{1}\left(1-\left|z_{1}\right|\right)}{1-\left|z_{1}\right|}
\end{aligned}
$$

where $r_{1}=\left|z_{1}\right|, r_{2}=\left|z_{2}\right|$.

Here we made use of

$$
\frac{1}{2 \pi i} \int_{\mathbb{T}} \frac{d z}{\zeta-z}=1 ; \int_{0}^{\pi} \frac{\omega_{2}\left(t_{2}\right) d t_{1}}{\left|e^{i t_{2}}-r_{2}\right|} \leq \text { const. }
$$

Repeating the arguments of Lemma 2.3 yields the desired assertion. Analogously we can obtain estimates (5) and (6).

From (2) and (3)-(5) we have

Corollary 3.2 There exist positive constants $C_{1}$ and $C_{2}$ depending only on $\omega$, such that

$$
C_{1} \max _{1 \leq k \leq n}\left\{\max _{1 \leq i_{1}<\ldots<i_{k} \leq n} \sup _{z \in \mathbb{U}^{n}}\left\{|g(z)|+\|f\|_{\infty}\right\}\right\} \leq
$$




$$
\|f\|_{\widetilde{\Lambda}(\omega)} \leq C_{2} \max _{1 \leq k \leq n}\left\{\max _{1 \leq i_{1}<\ldots<i_{k} \leq n} \sup _{z \in \mathbb{U}^{n}}\left\{|g(z)|+\|f\|_{\infty}\right\}\right\}
$$

where

$$
g(z)=\prod_{j=1}^{k} \frac{\omega_{i_{j}}\left(1-\left|z_{i_{j}}\right|\right)}{1-\left|z_{i_{j}}\right|} \cdot \frac{\partial^{k} f(z)}{\partial z_{i_{1}} \ldots \partial z_{i_{k}}} .
$$

Theorem 3.3 Suppose $f \in \mathbb{H}^{\infty}\left(\mathbb{U}^{n}\right)$. Then $f \in \widetilde{\Lambda}_{*}^{a}(\omega)$ if and only if

$$
\left|\frac{\partial^{2 k} f(z)}{\partial z_{i_{1}}^{2} \ldots z_{i_{k}}^{2}}\right| \leq C \cdot C_{(k)}(f) \cdot \prod_{j=1}^{k} \frac{\omega_{i_{j}}\left(1-\left|z_{i_{j}}\right|\right)}{\left(1-\left|z_{i_{j}}\right|\right)^{2}}
$$

where $1 \leq i_{k} \leq n, 1 \leq k \leq n$.

Theorem $3.4 f \in \widetilde{\Lambda}^{a}(\omega)$ if and only if $z_{j} \cdot f \in \widetilde{\Lambda}^{a}(\omega)$, for some $1 \leq j \leq n$.

Proof. Let $j=1$ and $z_{j} \cdot f \in \widetilde{\Lambda}^{a}(\omega)$. We need an estimate

$$
\left|\frac{\partial^{k} f(z)}{\partial z_{i_{1}} \ldots z_{i_{k}}}\right| \leq C \cdot C_{(k)}(f) \cdot \prod_{j=1}^{k} \frac{\omega_{i_{j}}\left(1-\left|z_{i_{j}}\right|\right)}{1-\left|z_{i_{j}}\right|} .
$$

In the case $i_{1} \neq 1$ it is evident.

Otherwise we have

$$
\frac{\partial^{k} f(z)}{\partial z_{i_{1}} \ldots \partial z_{i_{k}}}=\frac{1}{z_{1}} \cdot \frac{\partial^{k}\left(f(z) \cdot z_{1}\right)}{\partial z_{i_{1}} \ldots \partial z_{i_{k}}}-\frac{1}{z_{1}^{2}} \frac{\partial^{k}\left(f(z) \cdot z_{1}\right)}{\partial z_{i_{2}} \ldots \partial z_{i_{k}}} .
$$

Since $z_{1} \cdot f \in \widetilde{\Lambda}^{a}(\omega)$, we obtain

$$
\left|\frac{\partial^{k}\left(f(z) t_{1}\right)}{\partial t_{1} \ldots z_{i_{k}}}\right| \leq C \cdot C_{(k)}(f) \cdot \frac{\omega_{i_{j}}\left(1-\left|t_{1}\right|\right)}{1-\left|t_{1}\right|} \prod_{j=2}^{k} \frac{\omega_{i_{j}}\left(1-\left|z_{i_{j}}\right|\right)}{1-\left|z_{i_{j}}\right|}
$$

Integrating in $t_{1} \in\left[0, z_{1}\right]$ alond the radius, we obtain

$$
\left|\frac{\partial^{k-1} f(z)}{\partial z_{i_{2}} \ldots z_{i_{k}}}\right| \leq C_{i_{2} \cdot i_{k}}(f) \cdot \prod_{j=2}^{k} \frac{\omega_{i_{j}}\left(1-\left|z_{i_{j}}\right|\right)}{\left|z_{1}\right|\left(1-\left|z_{i_{j}}\right|\right)} .
$$

For $\left|z_{1}\right| \geq 1 / 2$ we have

$$
\left|\frac{\partial^{k-1} f(z)}{\partial z_{i_{2}} \ldots z_{i_{k}}}\right| \leq C_{i_{2} \cdot i_{k}}(f) \cdot \prod_{j=2}^{k} \frac{\omega_{i_{j}}\left(1-\left|z_{i_{j}}\right|\right)}{1-\left|z_{i_{j}}\right|} .
$$


On the other hand, the latter estimate for $\left|z_{1}\right| \leq 1 / 2$ is a consequence of the modules modules maximum principle. Talking into account (10) we get (9).

Conversely, let $f \in \widetilde{\Lambda}^{a}(\omega)$. We have for $i_{j} \neq 1, j=1, \ldots, n$

$$
\left|\frac{\partial^{k}\left(f(z) z_{1}\right)}{\partial z_{i_{1}} \ldots z_{i_{k}}}\right| \leq C \cdot C_{(k)}(f) \cdot \prod_{j=1}^{k} \frac{\omega_{i_{j}}\left(1-\left|z_{i_{j}}\right|\right)}{1-\left|z_{i_{j}}\right|} .
$$

Suppose $i_{1}=1$. We have

$$
\frac{\partial^{k}\left(f(z) z_{1}\right)}{\partial z_{i_{1}} \ldots z_{i_{k}}}=z_{1} \frac{\partial^{k} f(z)}{\partial z_{i_{1}} \ldots z_{i_{k}}}+\frac{\partial^{k-1} f(z)}{\partial z_{i_{2}} \ldots z_{i_{k}}}
$$

and

$$
\left|\frac{\partial^{k-1} f(z)}{\partial z_{i_{2}} \ldots z_{i_{k}}}\right| \leq C_{i_{2} \cdot i_{k}}(f) \cdot \prod_{j=2}^{k} \frac{\omega_{i_{j}}\left(1-\left|z_{i_{j}}\right|\right)}{1-\left|z_{i_{j}}\right|}
$$

This implies

$$
\left|\frac{\partial^{k}\left(f(z) z_{1}\right)}{\partial z_{i_{1}} \ldots z_{i_{k}}}\right| \leq C_{(k)}(f) \cdot \prod_{j=1}^{k} \frac{\omega_{i_{j}}\left(1-\left|z_{i_{j}}\right|\right)}{\left|z_{1}\right|\left(1-\left|z_{i_{j}}\right|\right)} .
$$

Note that the classes of S. M. Nikol'skii $\Lambda\left(\alpha_{1}, \ldots, \alpha_{n}\right)$ do not possess property of $\widetilde{\Lambda}^{a}(\omega)$, proved in Theorem 3.4. This can be checked by taking $f(z)=\phi\left(z_{1}\right)+\psi\left(z_{2}\right)$, where $\phi$ and $\psi$ belong to $\mathbb{H}^{\infty}$. For $\phi$ and $\psi$ and for $\alpha_{1}, \alpha_{2}>0$ we have $f \in \Lambda^{a}\left(\alpha_{1}, \alpha_{2}\right)$. But it is clear that the inclusion $z_{1} z_{2} f \in \Lambda^{a}\left(\alpha_{1}, \alpha_{2}\right)$ is not true for all possible $\phi$ and $\psi$.

Theorem 3.5 A function $f$ belongs to $\widetilde{\Lambda}_{*}^{a}(\omega)$ if and only if $z_{j}^{2} \cdot f \in \widetilde{\Lambda}_{*}^{a}(\omega)$, for some $1 \leq j \leq n$.

Theorem 3.6 $f \in \widetilde{\Lambda}^{a}(\omega)$ if and only if $D^{m} f \in \widetilde{\Lambda}^{a}(\widetilde{\omega}), \widetilde{\omega}_{j}(t)=\omega_{j}(t) / t^{m}, m=$ $\left(m_{1}, \ldots, m_{n}\right), m_{j}$ being nonnegativ integer, $1 \leq j \leq n$.

Before giving the proof, we show several lemmas.

Lemma 3.7 Let $\alpha=\left(\alpha_{1}, \ldots, \alpha_{n}\right)$, where $\alpha_{j}, 1 \leq j \leq n$ are some nonnegative integers. Then

$$
D^{\alpha} f(z)=\frac{1}{\alpha !} \frac{\partial^{\alpha}\left(f(z) z^{\alpha}\right)}{\partial z^{\alpha}}, z=\left(z_{1}, \ldots, z_{n}\right), \alpha !=\alpha_{1} ! \ldots \alpha_{n} !
$$

See [12] for a proof. 
Lemma 3.8 Let $h \in \mathbb{H}\left(\mathbb{U}^{n}\right)$ and $|h(z)| \leq \omega_{1}\left(1-\left|z_{1}\right|\right) \ldots \omega_{j}\left(1-\left|z_{j}\right|\right)$ $1 \leq j \leq n$. Then

$$
\left|\frac{\partial^{k_{1}+\ldots+k_{j}} h(z)}{\partial z_{1}^{k_{1}} \ldots z_{j}^{k_{j}}}\right| \leq \frac{\omega_{1}\left(1-\left|z_{1}\right|\right) \ldots \omega_{j}\left(1-\left|z_{j}\right|\right)}{\left(1-\left|z_{1}\right|\right)^{k_{1}} \ldots\left(1-\left|z_{j}\right|\right)^{k_{j}}}, 1 \leq j \leq n .
$$

Proof. Let

$$
\widetilde{T}_{j}=\left\{\zeta_{j}, \zeta_{j}=z_{j}+\eta\left(1-\left|z_{j}\right|\right) e^{i \theta_{j}}\right\}, j=1, \ldots, n
$$

Using the Cauchy formula for $\widetilde{T}^{j}=\widetilde{T}_{1} \times \ldots \times \widetilde{T}_{j}$, we obtain

$$
\frac{\partial^{k_{1}+\ldots+k_{j}} h(z)}{\partial z_{1}^{k_{1}} \ldots z_{j}^{k_{j}}}=\frac{1}{(2 \pi i)^{j}} \int_{T^{j}} \frac{\left.h\left(\zeta_{1}, \ldots, \zeta_{j}\right), z^{\prime}\right) d \zeta_{1} \ldots \zeta_{j}}{\left(\zeta_{1}-z_{1}\right)^{k_{1}+1} \ldots\left(\zeta_{j}-z_{j}\right)^{k_{j}+1}}
$$

for $z^{\prime}=\left(z_{j+1}, \ldots, z_{n}\right)$.

Hence,

$$
\begin{aligned}
\left|\frac{\partial^{k_{1}+\ldots+k_{j}} h(z)}{\partial z_{1}^{k_{1}} \ldots z_{j}^{k_{j}}}\right| & \leq \frac{1}{(2 \pi i)^{j}} \int_{-\pi}^{\pi} \ldots \int_{-\pi}^{\pi} \frac{\omega_{1}\left(1-\left|z_{1}\right|\right) \ldots \omega_{j}\left(1-\left|z_{j}\right|\right)}{\left(1-\left|z_{1}\right|\right)^{k_{1}} \ldots\left(1-\left|z_{j}\right|\right)^{k_{j}}} d \theta_{1} \ldots d \theta_{j} \\
& =\frac{\omega_{1}\left(1-\left|z_{1}\right|\right) \ldots \omega_{j}\left(1-\left|z_{j}\right|\right)}{\left(1-\left|z_{1}\right|\right)^{k_{1}} \ldots\left(1-\left|z_{j}\right|\right)^{k_{j}}}
\end{aligned}
$$

and the lemma follows.

Proof of Theorem. Let $f \in \widetilde{\Lambda}^{a}(\omega)$. Then $z^{m} f \in \widetilde{\Lambda}^{a}(\omega)$ and

$$
\left|\frac{\partial^{k}\left(f(z) z^{m}\right)}{\partial z_{i_{1}} \ldots \partial z_{i_{k}}}\right| \leq \frac{\omega_{i_{1}}\left(1-\left|z_{i_{1}}\right|\right) \ldots \omega_{i_{k}}\left(1-\left|z_{i_{k}}\right|\right)}{\left(1-\left|z_{i_{1}}\right|\right) \ldots\left(1-\left|z_{i_{k}}\right|\right)}
$$

From Lemma 3.6 it follows that

$$
\left|\frac{\partial^{m}\left(f(z) z^{m}\right)}{\partial z_{i_{1}}^{m_{1}} \ldots \partial z_{i_{k}}^{m_{k}}}\right| \leq \frac{\omega_{i_{1}}\left(1-\left|z_{i_{1}}\right|\right) \ldots \omega_{i_{k}}\left(1-\left|z_{i_{k}}\right|\right)}{\left(1-\left|z_{i_{1}}\right|\right)^{m_{i_{1}}+1} \ldots\left(1-\left|z_{i_{k}}\right|\right)^{m_{i_{k}}+1}} .
$$

Therefore, from Lemma 3.5 yields $D^{m} f \in \widetilde{\Lambda}^{a}(\widetilde{\omega})$.

Conversely, let $D^{m} f \in \widetilde{\Lambda}^{a}(\widetilde{\omega})$.

Then the estimation (12) holds. Integrating in $t_{1}, \ldots, t_{k}$ as in Theorem 3.4 , we arrive at (11).

Theorem $3.9 f \in \widetilde{\Lambda}_{*}^{a}(\omega)$ if and only if $D^{m+2} f \in \widetilde{\Lambda}_{*}^{a}(\widetilde{\omega}), \widetilde{\omega}_{j}(t)=\omega_{j}(t) / t^{m+2}, m=$ $\left(m_{1}, \ldots, m_{n}\right)$, where $m_{j}(1 \leq j \leq n)$ is nonnegativ integer.

Theorem 3.10 The classe $\widetilde{\Lambda}^{a}(\omega)$ forms an algebra. 
Proof. Let $f, g \in \widetilde{\Lambda}^{a}(\omega)$.

We prove that $f \cdot g \in \widetilde{\Lambda}^{a}(\omega)$. We have

$$
\frac{\partial^{k}(f \cdot g)}{\partial z_{i_{1}} \ldots \partial z_{i_{k}}}=\sum_{i_{l}=0}^{1} \frac{\partial^{l} f(z)}{\partial z_{i_{1}} \ldots \partial z_{i_{l}}} \cdot \frac{\partial^{k-1} g(z)}{\partial z_{i_{l+1}} \ldots \partial z_{i_{k}}} .
$$

Hence, we get by Theorem 2.1,

$$
\left|\frac{\partial^{l} f(z)}{\partial z_{i_{1}} \ldots \partial z_{i_{l}}}\right| \leq C_{(k, l)}(f) \prod_{j=1}^{l} \frac{\omega_{i_{j}}\left(1-\mid z_{i_{j}}\right)}{1-\left|z_{i_{j}}\right|}
$$

and

$$
\left|\frac{\partial^{k-l} g(z)}{\partial z_{i_{l+1}} \ldots \partial z_{i_{k}}}\right| \leq C_{(k, l)}(g) \prod_{j=l+1}^{k} \frac{\omega_{i_{j}}\left(1-\mid z_{i_{j}}\right)}{1-\left|z_{i_{j}}\right|}
$$

It follows easily that

$$
\left|\frac{\partial^{k}(f(z) g(z)}{\partial z_{i_{1}} \ldots \partial z_{i_{k}}}\right| \leq C_{(k)}(f g) \prod_{j=1}^{k} \frac{\omega_{i_{j}}\left(1-\mid z_{i_{j}}\right)}{1-\left|z_{i_{j}}\right|}
$$

Using Theorem 2.1 once again, we get $f \cdot g \in \widetilde{\Lambda}^{a}(\omega)$.

The remaining algebra properties are .

\section{Toeplitz Operators in classes $\widetilde{\Lambda}\left(\omega_{1}, \ldots, \omega_{n}\right)$}

Definition 4.1 The Toeplitz operator with a symbol $h \in \mathbb{L}^{1}\left(\mathbb{T}^{n}\right)$ is the integral operator

$$
\begin{aligned}
& T_{h}(f)(z)= \frac{1}{(2 \pi i)^{n}} \int_{\mathbb{T}^{n}} \frac{f(\zeta) h(\zeta)}{\zeta-z} d \zeta \\
& \equiv \frac{1}{(2 \pi i)^{n}} \int_{\mathbb{T}^{n}} \frac{f\left(\zeta_{1}, \ldots \zeta_{n}\right) h\left(\zeta_{1}, \ldots \zeta_{n}\right) d \zeta_{1} \cdots d \zeta_{n}}{\left(\zeta_{1}-z_{1}\right) \cdots\left(\zeta_{n}-z_{n}\right)}, \\
& \quad z=\left(z_{1}, \ldots, z_{n}\right) \in \mathbb{U}^{n} .
\end{aligned}
$$

Recall that a function $f$ is called a multiplicator of the space $X$, if $f \cdot g \in$ $X$ for any $g \in X$.

Theorem 4.2 Let $f \in \widetilde{\Lambda}^{a}(\omega)$ and let $h=h_{1}+\overline{h_{2}}$, where $h_{1}$ is a holomorphic multiplicator of $\widetilde{\Lambda}^{a}(\omega)\left(\omega=\left(\omega_{1}, \ldots, \omega_{n}\right), \omega_{j} \in \Omega, 1 \leq j \leq n\right)$, and $h_{2} \in \mathbb{H}^{\infty}\left(\mathbb{U}^{n}\right)$.

Then $T_{h}(f) \in \widetilde{\Lambda}^{a}(\omega)$. 
Proof. Obviously,

$$
\begin{aligned}
T_{h}(f)(z) & =\frac{1}{(2 \pi i)^{n}} \int_{\mathbb{T}^{n}} \frac{f(\zeta) h(\zeta)}{\zeta-z} d \zeta \\
& =f(z) h_{1}(z)+\frac{1}{(2 \pi i)^{n}} \int_{\mathbb{T}^{n}} \frac{f(\zeta) \overline{h_{2}(\zeta)}}{\zeta-z} d \zeta \\
& =f(z) h_{1}(z)+T_{\overline{h_{2}}}(f)(z) .
\end{aligned}
$$

Furthermore, $f \cdot h_{1} \in \widetilde{\Lambda}^{a}(\omega)$ since $h_{1}$ is a multiplicator of $\widetilde{\Lambda}^{a}(\omega)$. Therefore it suffices to prove an estimate of the form

$$
\left|\frac{\partial^{k} T_{\overline{h_{2}}}(f)(z)}{\partial z_{i_{1}} \cdots \partial z_{i_{k}}}\right| \leq C\left(f, h_{2}\right) \prod_{j=1}^{k} \frac{\omega\left(1-\left|z_{i j}\right|\right)}{\left.1-\left|z_{i j}\right|\right)}
$$

Without loss of generality we can assume that $\left(i_{1}, \ldots, i_{k}\right) \equiv(1, \ldots, k)$. Then a simple transformation gives

$$
\begin{aligned}
\frac{\partial^{k} T_{\overline{h_{2}}}(f)(z)}{\partial z_{1} \cdots \partial z_{k}} & =\frac{1}{(2 \pi i)^{n}} \int_{\mathbb{Q}^{n}} \frac{f\left(e^{i \vartheta}\right) \overline{h_{2}\left(e^{i \vartheta}\right)} d e^{i \vartheta}}{\prod_{j=1}^{k}\left(e^{i \vartheta_{j}}-r_{j} e^{i \varphi_{j}}\right)^{2} \prod_{j=k+1}^{n}\left(e^{i \vartheta_{j}}-r_{j} e^{i \varphi_{j}}\right)} \\
& =\frac{e^{-i \varphi_{k}^{\prime}}}{(2 \pi)^{n}} \int_{\mathbb{Q}^{n}} \frac{f\left(e^{i(\varphi+t)}\right) \overline{h_{2}\left(e^{i(\varphi+t)}\right)} e^{i t} d t}{\prod_{j=1}^{k}\left(e^{i t_{j}}-r_{j}\right)^{2} \prod_{j=k+1}^{n}\left(e^{i t_{j}}-r_{j}\right)}
\end{aligned}
$$

where $t=\left(t_{1}, \ldots, t_{n}\right), t_{j}=\vartheta_{j}-\varphi_{j}, \varphi_{k}^{\prime}=\left(\varphi_{1}, \ldots, \varphi_{k}\right), e^{i t}=e^{i t_{1}} \cdots e^{i t_{n}}$. Applying Lemma 2.4 we obtain the equalities

$$
\begin{aligned}
& \frac{\partial^{k} T_{\overline{h_{2}}}(f)(z)}{\partial z_{1} \cdots \partial z_{k}}=\frac{e^{-i \varphi_{k}^{\prime}}}{(2 \pi)^{n}} \int_{\mathbb{Q}^{n}} \frac{f\left(e^{i(\varphi+t)}\right) \overline{h_{2}\left(e^{i(\varphi+t)}\right)} e^{i t} d t}{\prod_{j=1}^{k}\left(e^{i t_{j}}-r_{j}\right)^{2} \prod_{j=k+1}^{n}\left(e^{i t_{j}}-r_{j}\right)}
\end{aligned}
$$

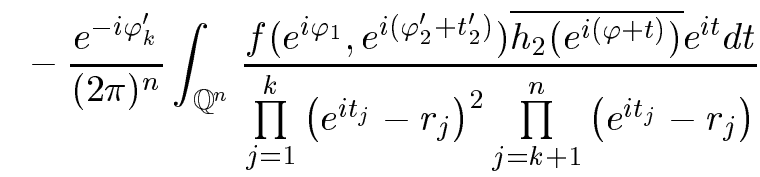

$$
\begin{aligned}
& =\frac{e^{-i \varphi_{k}^{\prime}}}{(2 \pi)^{n}} \int_{\mathbb{Q}^{n}} \frac{\Delta_{t_{1}} f\left(e^{i \varphi_{1}}, e^{i\left(\varphi_{2}^{\prime}+t_{2}^{\prime}\right)}\right) \overline{h_{2}\left(e^{i(\varphi+t)}\right)} e^{i t} d t}{\prod_{j=1}^{k}\left(e^{i t_{j}}-r_{j}\right)^{2} \prod_{j=k+1}^{n}\left(e^{i t_{j}}-r_{j}\right)} .
\end{aligned}
$$

For the first $k$ variables we have

$$
\frac{\partial^{k} T_{\overline{h_{2}}}(f)(z)}{\partial z_{1} \cdots \partial z_{k}}=\frac{e^{-i \varphi_{k}^{\prime}}}{(2 \pi)^{n}} \int_{\mathbb{Q}^{n}} \frac{\Delta_{t_{k} \ldots t_{1}} f\left(e^{i \varphi_{k}^{\prime}}, e^{i\left(\varphi_{k+1}^{\prime \prime}+t_{k+1}^{\prime \prime}\right)}\right)}{\prod_{j=1}^{k}\left(e^{i t_{j}}-r_{j}\right)^{2} \prod_{j=k+1}^{n}\left(e^{i t_{j}}-r_{j}\right)} \overline{h_{2}\left(e^{i(\varphi+t)}\right)} e^{i t} d t,
$$


where $\varphi_{k+1}^{\prime \prime}=\left(\varphi_{k+1}, \ldots, \varphi_{n}\right), t_{k+1}^{\prime \prime}=\left(t_{k+1}, \ldots, t_{n}\right)$.

Repeating the same argument gives

$$
\begin{aligned}
& \frac{\partial^{k} T_{\overline{h_{2}}}(f)(z)}{\partial z_{1} \cdots \partial z_{k}}=\frac{e^{-i \varphi_{k}^{\prime}}}{(2 \pi)^{n}} \int_{\mathbb{Q}^{n}} \frac{\Delta_{t_{n} \ldots t_{1}} f\left(e^{i \varphi}\right) \overline{h_{2}\left(e^{i(\varphi+t)}\right)} e^{i t} d t}{\prod_{j=1}^{k}\left(e^{i t_{j}}-r_{j}\right)^{2} \prod_{j=k+1}^{n}\left(e^{i t_{j}}-r_{j}\right)} \\
& +\frac{e^{-i \varphi_{k}^{\prime}}}{(2 \pi)^{n}}\left\{\int_{\mathbb{Q}^{n}} \frac{\Delta_{t_{k} \ldots t_{1}} f\left(e^{i \varphi_{k+1}^{\prime}}, e^{i\left(\varphi_{k+2}^{\prime \prime}+t_{k+2}^{\prime \prime}\right)} \overline{h_{2}\left(e^{i(\varphi+t)}\right)} e^{i t} d t\right.}{\prod_{j=1}^{k}\left(e^{i t_{j}}-r_{j}\right)^{2} \prod_{j=k+1}^{n}\left(e^{i t_{j}}-r_{j}\right)}\right. \\
& +\int_{\mathbb{Q}^{n}} \frac{\Delta_{t_{k+1} \ldots t_{1}} f\left(e^{i \varphi_{k+2}^{\prime}}, e^{i\left(\varphi_{k+3}^{\prime \prime}+t_{k+3}^{\prime \prime}\right)} \overline{h_{2}\left(e^{i(\varphi+t)}\right)} e^{i t} d t\right.}{\prod_{j=1}^{k}\left(e^{i t_{j}}-r_{j}\right)^{2} \prod_{j=k+1}^{n}\left(e^{i t_{j}}-r_{j}\right)}
\end{aligned}
$$

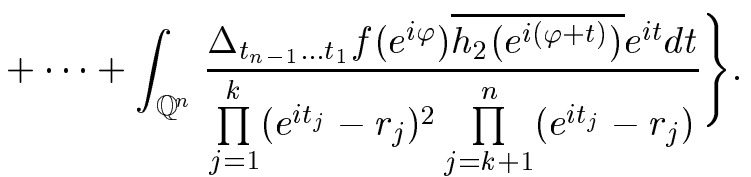

To transform the terms in figure brackets we denote first of them by $\mathcal{J}_{1, \ldots, k}^{1, \ldots, k+1} \equiv \mathcal{J}_{k}^{k+1}$, where the lower index means the lower index of $\Delta$ and the upper one is the number of free variables in $f\left(e^{i \varphi_{k+1}^{\prime}}, e^{i\left(\varphi_{k+2}^{\prime \prime}+t_{k+2}^{\prime \prime}\right)}\right)$. Then it is clear that

$$
\mathcal{J}_{k}^{k+1}=\mathcal{J}_{k}^{k+1}-\mathcal{J}_{k}^{k+2}+\mathcal{J}_{k}^{k+2}=\mathcal{J}_{k, k+2}^{k+2}+\mathcal{J}_{k}^{k+2} .
$$

Repeating the same argument we get

$$
\mathcal{J}_{k}^{k+1}=\mathcal{J}_{k, k+2, \ldots, n}+\mathcal{J}_{k, k+3, \ldots, n}+\ldots+\mathcal{J}_{k, n} .
$$

Similar representations are true for the remaining terms in brackets. On the other hand, it is clear that

$\left|\Delta_{t_{i_{1}} \ldots t_{i_{l}}} f\left(e^{i \varphi}\right)\right| \leq C_{l}(f) \omega\left(\left|t_{i_{1}}\right|\right) \cdots \omega\left(\left|t_{i_{l}}\right|\right.$ and $\left|h_{2}\left(e^{i(\varphi+t)}\right)\right| \leq$ const.

Thus, for obtaining the desired estimate one has to evaluate the integral

$$
I \equiv C_{f} \underbrace{\int_{-\pi}^{\pi} \cdots \int_{-\pi}^{\pi}}_{n} \frac{\omega\left(\left|t_{1}\right|\right) \cdots \omega\left(\left|t_{k}\right|\right) \prod^{*} \omega_{i_{j}}\left(\left|t_{i j}\right|\right)}{\prod_{j=1}^{k}\left|e^{i t_{j}}-r_{j}\right|^{2}}\left|d t_{j}\right| \cdots\left|d t_{n}\right|,
$$

where $\prod^{*}=\prod_{k<i_{j} \leq m}, m \leq n$. Using Lemma 2.3 and (7) we get

$$
I \leq C \cdot C_{k}(f h) \prod_{j=1}^{k} \frac{\omega_{j}\left(1-\left|z_{j}\right|\right)}{\left(1-\left|z_{j}\right|\right)}
$$


where $\left|z_{j}\right|=r_{j}, 1 \leq j \leq n$. Hence we come to the following estimate which proves our theorem

$$
\left|\frac{\partial^{k} T_{\overline{h_{2}}}(f)(z)}{\partial z_{1} \cdots \partial z_{k}}\right| \leq C \cdot C_{k}(f h) \prod_{j=1}^{k} \frac{\omega_{j}\left(1-\left|z_{j}\right|\right)}{1-\left|z_{j}\right|} .
$$

\section{Linear Continuous Functionals on $\mathbb{H}^{p}(\omega)$ $0<p \leq 1$ and Applications}

We denote by $S$ the class of nonpositive measurable functions $\omega$ on $(0,1)$, for which there are positive numbers $M_{\omega}, m_{\omega}, q_{\omega}\left(m_{\omega}, q_{\omega} \in(0,1)\right)$ exist, such that for all $r \in(0,1), \lambda \in\left[q_{\omega}, 1\right]$

$$
m_{\omega} \leq \frac{\omega(\lambda r)}{\omega(r)} \leq M_{\omega}
$$

We set

$$
\alpha_{\omega}=\log m_{\omega} / \log q_{\omega}, \quad \beta_{\omega}=\log M_{\omega} / \log \left(1 / q_{\omega}\right) .
$$

The properties of function from $S$ have been studied in [12]. From [12] and [9] we have $\Omega=S$ provided that $M_{\omega_{j}}<1$.

Let $\omega=\left(\omega_{1}, \cdots, \omega_{n}\right)$, where $\omega_{j} \in S, 1 \leq j \leq n$. We denote by $\mathbb{H}^{p}\left(\omega_{1}, \ldots, \omega_{n}\right)$ the class of functions $f$ holomorphic in $\mathbb{U}^{n}$, satisfying

$$
\int_{\mathbb{U}^{n}}\left|f\left(\zeta_{1}, \ldots, \zeta_{n}\right)\right|^{p} \prod_{j=1}^{n} \omega_{j}\left(1-\left|\zeta_{j}\right|\right) d m_{2 n}(\zeta)<+\infty,
$$

where $0<p \leq 1$ and $m_{2 n}(\zeta)$ is the $2 n$-dimensional Lebesgue measure in $\mathbb{U}^{n}$ (see [10]). These spaces are multidimensional generalisations of the wellknown classes of M. M. Djrbashian for $n=1, \omega(t)=t^{\alpha}, \alpha>-1$ (see[13]). Let $\omega_{j} \in S, j=1, \ldots, n$, and $0<p \leq 1$. A function $g \in \mathbb{H}\left(\mathbb{U}^{n}\right)$ is said to be in $\Lambda_{\omega}^{p}=\Lambda_{\omega_{1}, \ldots, \omega_{n}}^{p}$, if

$$
\|g\|_{\Lambda_{\alpha}^{p}}=\sup _{z \in \mathbb{U}^{n}}\left\{\left|D^{\alpha+1} g(z)\right| \cdot \frac{\omega(1-|z|)^{1 / p}}{(1-|z|)^{\alpha+2-2 / p}}\right\}<+\infty
$$

for all $\left.\alpha_{j}>\left(\alpha_{\omega_{j}}+2\right) / p, 1 \leq j \leq n\right)\left(\left|z_{j}\right| \rightarrow 1-0,1 \leq j \leq n\right)$.

The class $\Lambda_{\omega}^{p}$ arises in describing continuous linear functionals in the spaces of holomorphic functions with $\mathbb{L}^{p}$ metric (see [9], [14], [15]).

To characterize the dual space $\left(\mathbb{H}^{p}(.)\right)^{*}$ in terms of $\widetilde{\Lambda}^{a}($.$) , we first find a$ relation between the spaces $\widetilde{\Lambda}^{a}($.$) and \Lambda^{p}($.$) .$

For $f \in \Lambda^{p}(\alpha)$ and $m_{j}-1>\left(\alpha_{\omega_{j}}+2\right) / p(1 \leq j \leq n)$ we have 


$$
\left|D^{m} g(z)\right| \leq c \cdot \frac{(\omega(1-|z|))^{1 / p}}{(1-|z|)^{m+1-2 / p}}
$$

Then, $z^{m} \cdot g \in \widetilde{\Lambda}^{a}(\widetilde{\omega}), \widetilde{\omega}(t)=\omega^{1 / p}(t) t^{2 / p}$.

By Theorem 3.4 we get $g \in \widetilde{\Lambda}^{a}(\widetilde{\omega})$. It follows from Lemma 1.4 that

$$
\left|\frac{\partial^{m_{i_{1}}+\ldots+m_{i_{k}}} g(z)}{\partial z_{i_{1}}^{m_{i_{1}}} \partial z_{i_{k}}^{m_{i_{k}}}}\right| \leq C\|f\|_{\Lambda_{\omega}^{p}} \prod_{j=1}^{k} \frac{\left(1-\left|z_{j}\right|\right)^{m_{j}+1-2 / p}}{\left(\omega_{j}\left(1-\left|z_{j}\right|\right)\right)^{1 / p}}
$$

Hence

$$
\prod_{j=1}^{k} \frac{\left(\omega_{j}\left(1-\left|z_{j}\right|\right)\right)^{1 / p}}{\left(1-\left|z_{j}\right|\right)^{m_{j}+1-2 / p}}\left|\frac{\partial^{m_{i_{1}}+\ldots+m_{i_{k}}} g(z)}{\partial z_{i_{1}}^{m_{i_{1}}} \partial z_{i_{k}}^{m_{i_{k}}}}\right| \leq C_{1}\|f\|_{\Lambda_{\omega}^{p}}
$$

and

$$
C\|g\|_{\Lambda_{\omega}^{p}} \geq\|g\|_{\widetilde{\Lambda}^{a}(\widetilde{\omega})} .
$$

Now we estabilish the converse inequality.

Let $g \in \widetilde{\Lambda}^{a}(\widetilde{\omega})$. Then $z^{m} \cdot g \in \widetilde{\Lambda}^{a}(\widetilde{\omega})$. We have

$$
\left|D^{m}\left(f(z) z^{m}\right)\right| \leq C_{2}\|g\|_{\widetilde{\Lambda}^{a}(\widetilde{\omega})} \prod_{j=1}^{n} \frac{\left(1-\left|z_{j}\right|\right)^{m_{j}+1-2 / p}}{\left(\omega_{j}\left(1-\left|z_{j}\right|\right)\right)^{1 / p}} .
$$

Therefore

$$
\mid D^{m}\left(f(z) z^{m} \mid \frac{\left(\omega_{j}\left(1-\left|z_{j}\right|\right)\right)^{1 / p}}{\left(1-\left|z_{j}\right|\right)^{m_{j}+1-2 / p}} \leq C_{2}\|g\|_{\widetilde{\Lambda}^{a}(\widetilde{\omega})} .\right.
$$

We have proved the following

Theorem 5.1 A function $g \in \mathbb{H}\left(\mathbb{U}^{n}\right)$ belongs to $\Lambda^{p}(\omega)$ if and only if $g \in \Lambda^{a}(\widetilde{\omega})$ where $\widetilde{\omega}(t)=\omega^{1 / p}(t) t^{2 / p}$.

Now, using Theorem 6 from [11], we can describe the dual space $\left(\mathbb{H}^{p}(\alpha)\right)^{*}$ in terms of $\widetilde{\Lambda}^{a}(\widetilde{\omega})$.

Theorem 5.2 Let $\Phi$ be a continuous linear functional on $\mathbb{H}^{p}(\omega)$,

$\left(\omega_{j} \in \Omega, 1 \leq j \leq n\right)$, and let $g(z)=\Phi\left((1-z w)^{-1}\right)$

$\left(z, w \in \mathbb{U}^{n}\right)$. Then:

(i) (a) $g \in \widetilde{\Lambda}^{a}(\widetilde{\omega}), \quad \widetilde{\omega}(t)=\omega^{1 / p}(t) t^{2 / p}$.

(b) The functional $\Phi$ is representable in the form

$$
\Phi(f)=\lim _{\rho \rightarrow 1-0} \frac{1}{(2 \pi)^{n}} \int_{\mathbb{T}^{n}} f(\rho \zeta) g(\rho \bar{\zeta}) d m_{n}(\zeta),
$$


moreover, for some positive constants $C_{1}(\omega)$ and $C_{2}(\omega)$ we have

$$
C_{1}(\rho)\|\Phi\| \leq\|g\|_{\widetilde{\Lambda}^{a}(\widetilde{\omega})} \leq C_{2}(\rho)\|\Phi\| .
$$

(ii) Conversely, any function $g \in \widetilde{\Lambda}^{a}\left(\gamma_{1}, \ldots, \gamma_{n}\right)$ induces by (13) a continuous linear functional on $\mathbb{H}^{p}(\omega)$, which satisfies (14).

Now we turn to some applications of our results to division theorems in spaces $\mathbb{H}^{p}\left(\alpha_{1}, \ldots, \alpha_{n}\right)$ and $\widetilde{\Lambda}^{a}\left(\omega_{1}, \ldots, \omega_{n}\right)$. To this end, we need the following well-known definitions.

Definition 5.3 A function $g \in \mathbb{H}^{\infty}\left(\mathbb{U}^{n}\right)$ is called an inner function, if its radial boundary values satisfy $\left|g^{*}(w)\right|=1$ almost everywhere on $\mathbb{T}^{n}$.

Definition 5.4 An inner function $g \in \mathbb{H}^{\infty}\left(\mathbb{U}^{n}\right)$ is said to be good, if $u[g]=0$, where $u[g]$ is the least n-harmonic majorant of $\log |g|$ in $\mathbb{U}^{n}([10])$.

Definition 5.5 A function $h$ summable in $\mathbb{T}^{n}$ is said to be of the class $R L$, if its Fourier coefficients vanish outside the set $\mathbb{Z}_{+}^{n} \cup\left(-\mathbb{Z}_{+}^{n}\right)$.

In [16] the following theorem is proved.

Theorem 5.6 Let $h \in R L, 0<p \leq 1$. Then the following statements are equivalent:

(i) $T_{h}\left(\mathbb{H}^{p}\left(\alpha_{1}, \ldots, \alpha_{n}\right)\right) \subseteq \mathbb{H}^{p}\left(\alpha_{1}, \ldots, \alpha_{n}\right)$,

(ii) $h=h_{1}+\overline{h_{2}}$, where $h_{1} \in H^{\infty}\left(U^{n}\right)$ and $h_{2} \in\left(\mathbb{H}^{p}\left(\alpha_{1}, \ldots, \alpha_{n}\right)\right)^{*}$.

Theorem 5.7 Let $\alpha=\left(\alpha_{1}, \ldots, \alpha_{n}\right), \omega=\left(\omega_{1}, \ldots, \omega_{n}\right)\left(\alpha_{j}>-1,1 \leq\right.$ $j \leq n, \omega_{j} \in \Omega, j=1, \ldots, n$, and let $\mathbb{X}$ means olse of the classes $\mathbb{H}^{p}\left(\alpha_{1}, \ldots, \alpha_{n}\right)$ and $\widetilde{\Lambda}^{a}\left(\omega_{1}, \ldots, \omega_{n}\right)$. Further, let $f \in \mathbb{X}, \mathcal{J}$ be a good inner function, let $F \in \mathbb{H}^{\infty}\left(\mathbb{U}^{n}\right)$, and let $f=F \cdot \mathcal{J}$. Then $F \in \mathbb{X}$.

Proof. It is evident that

$T_{\overline{\mathcal{J}}}(f)(z)=\frac{1}{(2 \pi i)^{n}} \int_{T^{n}} \frac{f(\zeta) \overline{\mathcal{J}(\zeta)}}{\zeta-z} d \zeta=\frac{1}{(2 \pi i)^{n}} \int_{T^{n}} \frac{f(\zeta) d \zeta}{\mathcal{J}(\zeta)(\zeta-z)}=\frac{1}{(2 \pi i)^{n}} \int_{T^{n}} \frac{F(\zeta) d \zeta}{(\zeta-z)}$.

On the other hand, since $F$ is holomorphic it is representable by the Cauchy formula. Hence $F \in \mathbb{X}$ by Theorems 3.2 and 4.2. Thus, the quotient of $f \in \mathbb{X}$ and a good inner function belongs to $\mathbb{X}$.

\section{REFERENCES}

1. Korenblum, B. I.,On an extremal property of outer functions [in Russian], Mat. Zametki, vol. 10, no 1, pp. 53 - 68 (1971).

2. Havin, V. P., On factorization of functions analytic in the disk, smooth up to its boundary [in Russian], Zap. Nauch. Sem. LOMI, vol. 22, pp. $202-205$ (1971). 
3. Shamoian, F. A., Division by inner functions in some spaces of functions analytic in the disk [in Russian], Zap. Nauch. Sem. LOMI, vol. 22, pp. $206-208$ (1971).

4. Kahane, J. P., Best approximation in $L^{1}(T)$. Bull. Amer. Math. Soc., vol. 80, no 5, pp. 788-804, (1974).

5. Shamoian, F. A., Toeplitz operators and dividing on inner function in some spaces of analitic functions[in Russian]. Dokladi Akad.Nauk Arm.SSR, Mathematika, vol. 76, no 3, pp 109-113, (1983).

6. Yorikke, B., A multidimensional analogy of Privalov's theorem. Math.Nachrichten, vol. 107, pp. 221-233, (1982).

7. Rudin, W., Theory of functions in the unit ball in $\mathbb{C}^{n}$, Mir, Moscow (1984).

8. Shamoian, F. A., Harutyunyan A. V., On anisotropic spaces of functions holomorphic in the polydisk, bounded up to its boundary, $J$. of Contemp. Math. An., National Ac. of Sci. of Armenia, vol. 28, no. 6, pp. $50-68$ (1993).

9. Nikol'ski, S. M., Approximation of functions of several variables and embedding theorems [in Russian], Nauka, Moscow (1969).

10. Shamoian, F. A., Diagonal mappings and representability questions in some classes of functions holomorphic in the polydisk [in Russian], Sib. Math. J. vol. 31, no 2, pp. $197-215$ (1990).

11. Zygmund A., Trigonometric Series, Cambrige University Press, (1959)

12. Shamoian, F. A., Harutyunyan A. V., Toeplitz operators in multidimensional spa- ces $H^{p}(\alpha)$ of M.M.Djrbashian, J. of Contemp. Math. An., National Ac. of Sci. of Armenia, vol. 30, no. 2, pp. $70-78$ (1995)

13. Seneta, E. Functions of regular variation[in Russian], Nauka,Moscow (1985)

14. Djrbashian, M. M., On representability of some classes of functions meromorphic in the unit disk [in Russian], DAN of Armenia, vol. 3, no 1 , pp. $3-9$ (1945).

15. Duren, P., Romberg, A., Shields A., Linear funktionals of $H^{p}$ spaces with $0<p<1$, J.Reine Angew.Math., vol. 238, pp.32-60,(1969)

16. Frazer A. P., The dual space of $H^{p}$ of the polydisk for $0<p<1$, Duke Math. J., vol. 39, no 2, pp. 369-379, (1972)

17. Rudin, W., Theory of functions in the polydisk, Mir, Moscow (1974).

(Anahit Harutyunyan) Yerewan State University, Department of Informatics and Applied Mathematics, Alek Manukian 1, 375025 Yerewan, Armenia

E-mail address:anahit@ysu.am 